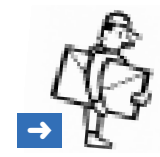

\section{Contrôles de qualité du laboratoire: praticiens, la QUALAB vous réserve des surprises pour tout bientôt!}

Tous ceux d'entre nous qui disposons d'un laboratoire au cabinet allons être soumis à de nouvelles directives élaborées par la Commission suisse pour l'assurance de qualité dans le laboratoire médical (QUALAB) pour le contrôle de qualité interne de nos laboratoires. Ces directives publiées le $1^{\text {er }}$ juillet 2005 sont présentées sur la page www.qualab.ch/CQI.htm. Elles sont soumises à critiques jusqu'au 31 juin 2006, avant qu'une version définitive ne soit produite. Je vous engage fortement à les lire. Comme vous le verrez, ces directives entraînent des surcoûts très conséquents pour les cabinets, une fréquence de contrôles inacceptable et une charge administrative majeure.

Ce projet de directives paraît totalement méconnu de la majorité des intéressés, à savoir les médecins praticiens disposant d'un laboratoire. C'est la raison pour laquelle, après en avoir été informé lors du dernier congrès annuel de la Société suisse d'allergologie et d'immunologie, je porte ceci à votre connaissance afin que vous puissiez réagir en connaissance de cause, individuellement ou en groupe, auprès de la QUALAB avant le 30 juin 2006, date de la fin de la période de «mise à l'enquête». Je suggère à toutes les sociétés des disciplines concernées de prendre position officiellement auprès de cette commission pour exiger que le projet soit revu et rendu conforme à la réalité de la médecine de cabinet, une réalité qui paraît très éloignée des préoccupations de cette commission.

Ci-dessous la lettre de commentaires que j'ai adressée à la QUALAB, détaillant mes préoccupations.

Cette lettre, adressée électroniquement à la QUALAB le 13 avril 2006, a déjà été diffusée électroniquement aux médecins affiliés à medromands.

\section{Messieurs,}

Nul ne remet en question les contrôles de qualité pour le laboratoire du cabinet médical auxquels nous sommes déjà soumis par le biais des contrôles externes. Par contre le projet de contrôle de qualité interne que vous proposez dépasse l'entendement. La lourdeur administrative et les coûts supplémentaires qui en résulteraient seraient tout à fait inacceptables et mettraient une fois de plus en danger la viabilité du laboratoire du cabinet médical, déjà sérieusement menacé par l'abaissement du tarif.

Demander un contrôle interne au minimum chaque 15 jours pour l'ensemble des paramètres analysés par un système analytique simple, tel que défini au point 4.2.1, relève de l'utopie absolue ou d'une volonté affichée de faire disparaître les laboratoires de cabinet. L'inutilité de contrôles aussi fréquents m'a été confirmée par le producteur d'appareil à chimie sèche que j'utilise (Cobas Ready), dont la fiabilité et la stabilité est confirmée de longue date par les contrôles externes trimestriels. Lorsque des contrôles réguliers sont préconisés par le fabricant, comme c'est le cas pour l'hématologie, la plupart d'entre nous s'astreint déjà aux contrôles quotidiens requis. Relever tous les paramètres sur des formulaires ad hoc relève de la tracasserie administrative la plus pure. Le coût de tels contrôles est par ailleurs prohibitif:

Pour les 18 paramètres de chimie que j'analyse, le coût supplémentaire estimé en fonction des chiffres donnés par le fournisseur de mon appareil sera approximativement de 2700.- CHF /an, travail de l'assistante compris. A quoi il faut rajouter le coût des contrôles de crase, d'hématologie et d'immunologie. Je ne suis pas à même évidemment d'estimer les coûts administratifs à la charge du contribuable pour que la Qualab puisse effectuer le contrôle des contrôles mais c'est certainement considérable.

Bien que nous vivions une époque où l'on $\mathrm{a}$, dans de multiples domaines, l'ambition folle et aberrante d'arriver au risque zéro, il faut avoir la sagesse de démontrer que les mesures que l'on propose, les coûts que l'on engage sont incontestablement utiles, ce qui n'est absolument pas le cas ici. Qui a démontré que les contrôles internes chaque 15 jours au cabinet permettaient de soigner mieux, de diminuer la morbidité et la mortalité? J'aimerais voir les études qui le prouvent; qui plus est, il nous faudrait des études pour chacun des appareils disponibles sur le marché suisse.

Le laboratoire du cabinet dispose d'un élément capital que les grands laboratoires n'ont pas: l'interprétation du résultat par le clinicien en fonction de la clinique et des analyses passées du patient. Lorsqu'une analyse diffère de ce qui est attendu, elle peut être contrôlée ponctuellement en interne ou en externe. C'est beaucoup plus ciblé et fiable que des screenings réalisés sans discernement chaque 15 jours, qui n'empêcheront pas, une fois ou l'autre, 
d'avoir un problème technique entre deux contrôles. En santé publique on connaît bien le fait que si la prévalence d'un problème dans la population est très faible, le dépistage va coûter trop cher pour le bénéfice que l'on en retire. Avez-vous démontré que la prévalence des problèmes consécutifs à des erreurs de laboratoire du praticien est élevée et qu'elle peut être réduite par un dépistage systématique? Sans ces données les mesures que vous préconisez sont dénuées de tout fondement.

Chaque jour nous autres cliniciens devons résister à la tentation facile de «tout faire pour exclure» et appliquer une démarche rigoureuse "evidence-based». J'aimerais être certain que ceux qui ont établi ce programme de contrôle interne l'ont fait avec la même rigueur et peuvent nous donner des chiffres précis sur les problèmes qui sont à l'origine de ce programme. J'aimerais être convaincu que l'on ne cède pas dans ce pays à la "contrôlite» abusive sous des prétextes assez éloignés de la santé de nos patients. En attendant il n'y a aucune raison de faire autre chose que ce que nous faisons actuellement, à savoir: nous soumettre à des contrôles externes, faire les contrôles internes requis par les constructeurs d'appareils et surtout avoir un regard critique sur tous les résultats qui sortent de nos laboratoires.

Dr L.-F. Debétaz, Lausanne

\section{Réplique}

La QUALAB formule ses directives sur la base de divers textes qui pour certains sont promulgués par le Département fédéral de l'intérieur, telle la Liste fédérale des analyses, alors que d'autres découlent de contrats signés par les partenaires. C'est en raison des obligations qui découlent de ces textes que la QUALAB a donné un mandat à un groupe de travail de rédiger les «directives pour le contrôle de qualité interne» actuellement sous le feu de la critique. La FMH était représentée dans ce groupe par un expert reconnu dans le domaine du laboratoire.

Il est important de noter que la version actuelle, publiée le $1^{\text {er }}$ juillet 2005, est une version initiale dont la teneur sera révisée en fonction des remarques que formuleront les personnes intéressées. Tous les commentaires peuvent être envoyés à l'adresse e-mail: qualint@qualab.ch jusqu'au 30 juin 2006. Il en sera tenu compte dans une future version de ces directives restant à rédiger.

Tous les participants aux contrôles de qualité externes ont directement été informés de l'existence de ces directives par ailleurs publiées sur le site de la QUALAB (www.qualab.ch).

Dr Franco Muggli, membre du Comité central de la FMH 